\title{
CSRR Loaded 2×1 Triangular MIMO Antenna for LTE Band Operation
}

\author{
A. Christina Josephine Malathi ${ }^{1}$, D. Thiripurasundari ${ }^{2}$ \\ ${ }^{1,2}$ School of Electronics and Communication Engineering, Vellore Institute of Technology, Vellore, India \\ *corresponding author, E-mail: achristina@vit.ac.in
}

\begin{abstract}
A Compact $2 \times 1$ multiple input multiple output (MIMO) antenna system is designed to operate in the LTE band 7 $(2.5-2.57) \mathrm{GHz}$. The proposed antenna consists of two triangular patches fed using microstrip line. In this work, complementary split ring resonator (CSRR) is loaded in the ground plane. The unloaded triangular patch antenna resonates at $5 \mathrm{GHz}$; whereas after loading it with CSRR, the same antenna resonates at $2.5 \mathrm{GHz}$. Size reductions of $72 \%$ compared to conventional patch antenna is obtained after the inclusion of CSRR in the ground plane. The designed antenna covers a bandwidth of (2.42 to 2.57) $\mathrm{GHz}$ with a maximum return loss of $-26.5 \mathrm{~dB}$ at $2.5 \mathrm{GHz}$ and isolation of $-17.3 \mathrm{~dB}$ between the ports with close inter element spacing of $0.17 \lambda$. The simulated correlation co-efficient is 0.0212 and the total active reflection co-efficient is 0.56 at the resonating frequency.
\end{abstract}

\section{Introduction}

In wireless communication systems, long term evolution (LTE) represents the fourth generation solution providing higher throughput, wider bandwidth and improved handoff capabilities compared to third generation networks. LTE wireless devices are expected to operate over different frequency bands in the range from $400 \mathrm{MHz}$ up to $4 \mathrm{GHz}$ [1, 2]. LTE uses MIMO technology to improve the efficiency of using radio spectrum. MIMO antenna is expected to be a key element to support LTE systems. MIMO depends on the use of multiple antennas on the transmitting and receiving sides thereby increasing the channel capacity without the need of additional bandwidth or power. There is an increasing demand for making new MIMO antenna systems that are compact and compatible with user terminals and other wireless portable devices. The integration of multiple antennas on the user mobile terminals is a design challenge that has been given considerable attention by researchers, due to the inherent size and inter-antenna coupling limitations. Hence it is important to make antennas that have enhanced channel capacity, bandwidth, gain, and diversity performance. These requirements make the design of MIMO antenna systems challenging.
There are a number of techniques available in the literature for isolation [3] and antenna miniaturization. Material loading is to use a substrate with high relative permittivity or loading high permittivity bar on a low permittivity substrate. As the length and width of the patch are inversely proportional to the square root of $\varepsilon_{r}$, use of high permittivity substrate results in miniaturization at the cost of reduced efficiency and lower bandwidth due to increased surface wave excitation within the substrate [4]. Loading of high dielectric substrate requires expensive material. Miniaturization up to four times can be achieved by reshaping the antenna by using fractal antenna or by cutting slots on the patch. This method suffers from high ohmic losses leading to low radiation efficiency with complex geometry and poor polarization purity $[5,6]$. Miniaturization up to four times can be achieved by folding the antenna and by using shorting posts. This technique suffers from decreased directivity and gain in addition to complex antenna geometry $[7,8]$. By introducing slots, the current path within the patch area is increased lowering the resonant frequency leading to $40-75 \%$ of side reduction. It provides wide bandwidth, but affects the radiation characteristics and provides poor polarization purity [9]. By introducing defects or slots in the ground plane, size reduction up to eight times is achieved but with lower efficiency, increased back lobe level and narrow bandwidth $[10,11]$.

Metamaterials such as ENG, MNG or DNG inspired antennas provide high degree of miniaturization with limited bandwidth, low efficiency and complex geometry [12-17]. Metamaterials (MTM) are also used for isolation enhancement between adjacent elements due to the presence of a band gap in their frequency response [18, 19]. In this paper, a novel design of compact $2 \times 1$ (two-element) MIMO patch with two identical triangular patch antennas with CSRRs in the ground plane is proposed. The operating band of the proposed antenna is the LTE band 7 with a resonant frequency of the antenna elements centered at 2.5 $\mathrm{GHz} .72 \%$ reduction in the size of the individual patch is achieved through CSRR loading, thus allowing the accommodation of the two patch antennas in an area of $50 \times 50 \mathrm{~mm}^{2}$ with $10 \mathrm{~mm}$ spacing between them. The total size of the proposed MIMO antenna system board is 50 $\times 50 \times 0.8 \mathrm{~mm}^{3}$. The paper is structured as follows. Section 2 discusses the design of antenna. Section 3 presents and 
associates the simulation and measured results and Section 4 concludes the paper.

\section{Design of the Antenna}

The triangular patch is etched on FR4 substrate with $\varepsilon_{\mathrm{r}}=4.4$ and thickness $\mathrm{t}=0.8 \mathrm{~mm}$. The dimensions of the patch antenna were chosen in such way that when two of such elements were duplicated for MIMO antenna design, they fit well within a $50 \times 50 \mathrm{~mm}^{2}$ area with a $10 \mathrm{~mm}$ gap between the elements. Patch of area $18.5 \times 16 \mathrm{~mm}^{2}$ was selected. Such a patch resonates at $5 \mathrm{GHz}$ in normal operating mode. To reduce the resonant frequency of the patch antenna, a single CSRR was etched out from its ground plane at its center.

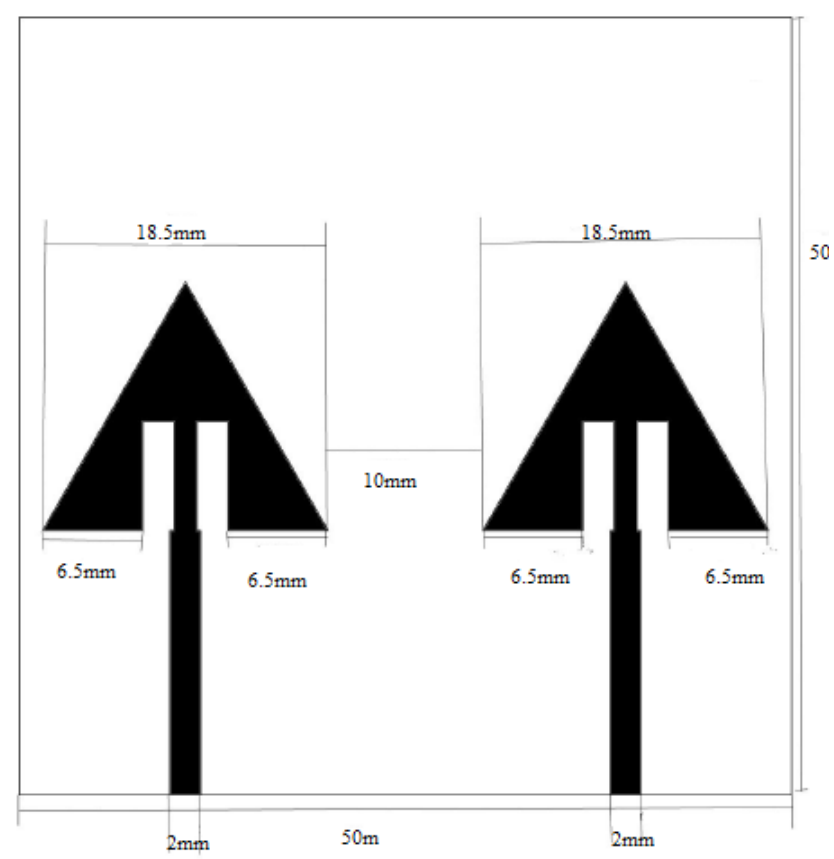

Figure 1: Geometry of the MIMO CSRR loaded patch antenna (a) top view

In the design of the single patch, the dimension of the CSRR was varied and the antenna was tuned to resonate at $2.5 \mathrm{GHz}$ using the commercial software HFSS. A two element MIMO antenna system was made using the same patch design covering an area of $50 \times 50 \times 0.8 \mathrm{~mm}^{3}$ with spacing of 10 $\mathrm{mm}$. The top and bottom layers of the proposed design are shown in Fig. 1(a), (b). The outer radius ' $r$ ' of the CSRR was $8.3 \mathrm{~mm}$, the width ' $w$ ' of each ring was $0.3 \mathrm{~mm}$, the spacing ' $s$ ' between the inner and outer ring was $0.3 \mathrm{~mm}$, and the slit ' $g$ ' in each ring was $0.5 \mathrm{~mm}$ which is shown in Fig.1 (b). The feed line width was $2 \mathrm{~mm}$, which gives a characteristic impedance of $50 \Omega$. The CSRR is a resonant structure which behaves as an LC tank circuit [20]. CSRR underneath the patch in the ground plane interacts with the electric field and provides effective negative permittivity and makes the patch act as band pass filter at the resonant frequency.

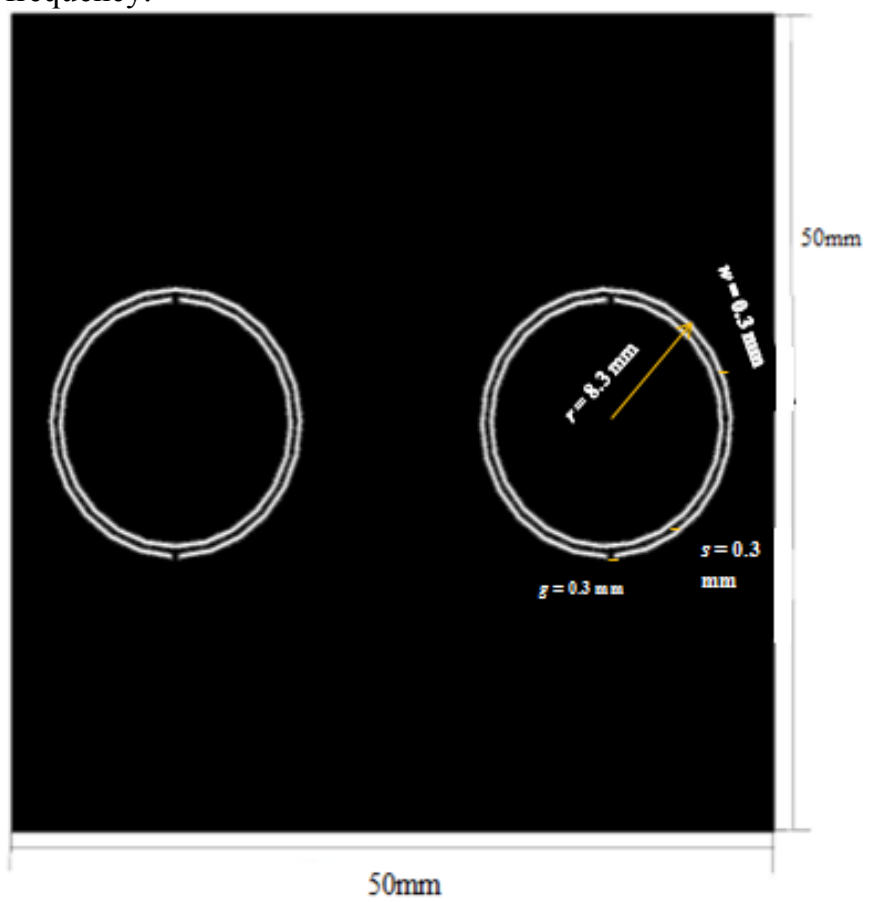

Figure 1: Geometry of the MIMO CSRR loaded patch antenna (b) bottom view

CSRR changes the characteristics of antenna cavity, and thus its resonance frequency is shifted. The antenna without CSRR resonated at $5 \mathrm{GHz}$ and with CSRR resonated at 2.5 $\mathrm{GHz}$ providing a miniaturization of $72 \%$. The dimensions of the antenna designed for $5 \mathrm{GHz}$ is $16 \times 18.5 \mathrm{~mm}^{2}$ and the dimensions of the antenna for $2.5 \mathrm{GHz}$ would be $29 \times 36$ $\mathrm{mm}^{2}$, hence providing miniaturization.

Nicolson Ross Weir had demonstrated the extraction of relative permittivity and permeability using $\mathrm{S}$ parameters [21-23] and is given by,

$$
\begin{gathered}
V_{1}=S_{11}+S_{21}---(1) \\
V_{2}=S_{21}-S_{11}---(2) \\
\varepsilon_{r}=\frac{2 c\left(1-V_{1}\right)}{\omega \operatorname{di}\left(1+V_{1}\right)}---(3) \\
\mu_{r}=\frac{2 c\left(1-V_{2}\right)}{\omega \operatorname{di}\left(1+V_{2}\right)}--(4)
\end{gathered}
$$

Where $\omega$ is the frequency in radian, $c$ is the velocity of light in $\mathrm{m} / \mathrm{s}, d$ is the thickness of the substrate, $i$ correspond to imaginary part and $V_{1}$ is the voltage maxima and $V_{2}$ is the voltage minima.

To study the effect of CSRR on MIMO antennas, permittivity and permeability are computed using Nicolson Ross Weir technique and both are negative.

The plot is done using equations (1-4) and is shown in Fig 2 for the antenna structure clearly explaining the concept of band pass filter at the resonant frequency of $2.5 \mathrm{GHz}$. 
Negative values of permittivity and permeability is the characteristic for band pass filter.

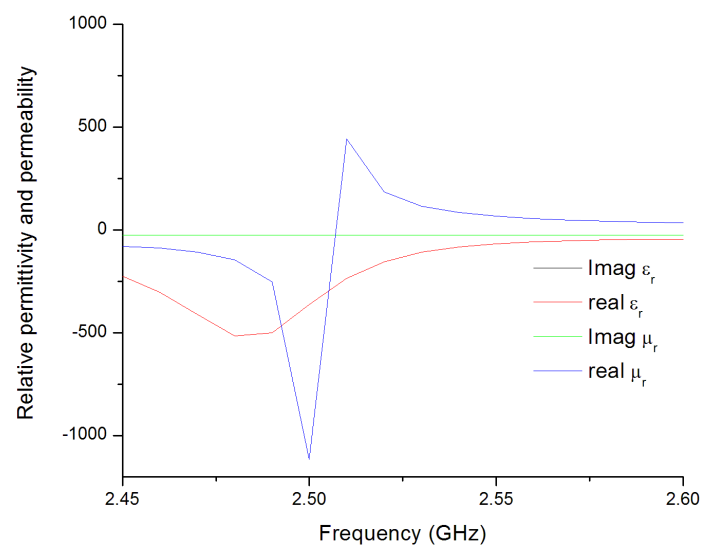

Figure 2: Relative permittivity and relative permeability vs. frequency for the antenna with CSRR

\section{Results and Discussion}

The design of CSRR is discussed followed by the return loss characteristics for single patch antenna and the return loss and isolation characteristics of MIMO antenna. Also the MIMO parameters such as ECC, TARC and gain are discussed for MIMO antenna in the following subsections.

\subsection{Effect of CSRR Design Parameters}

While tuning the antenna to resonate at $2.5 \mathrm{GHz}$, the dimensions of the CSRR were changed. These dimensions included the radius ' $r$ 'of the outer ring, the width ' $w$ ' of each ring, the spacing ' $s$ ' between the inner and outer ring and the slit ' $g$ ' in each ring. Slits do not have much effect on the resonant frequency. Thus, the width of the slits was kept at $0.5 \mathrm{~mm}$. As the radius of the CSRR, was increased, the resonant frequency of the antenna decreased. Increasing the width of the rings $w$ and the spacing between the two rings $s$ resulted in an increase in the resonant frequency of the antenna. Thus, by changing these parameters, the antenna was tuned at $2.5 \mathrm{GHz}$.

\subsection{Single Patch Reflection coefficient with and without CSRR}

The reflection co-efficient $(\mathrm{dB})$ for the antenna without CSRR is $-14 \mathrm{~dB}$ around $5 \mathrm{GHz}$ and with CSRR loaded patch is $-28 \mathrm{~dB}$ as shown in Fig. 3. The antenna resonates around $2.5 \mathrm{GHz}$ with a $10 \mathrm{~dB}$ bandwidth of approximately $50 \mathrm{MHz}$, clearly indicating the miniaturization.

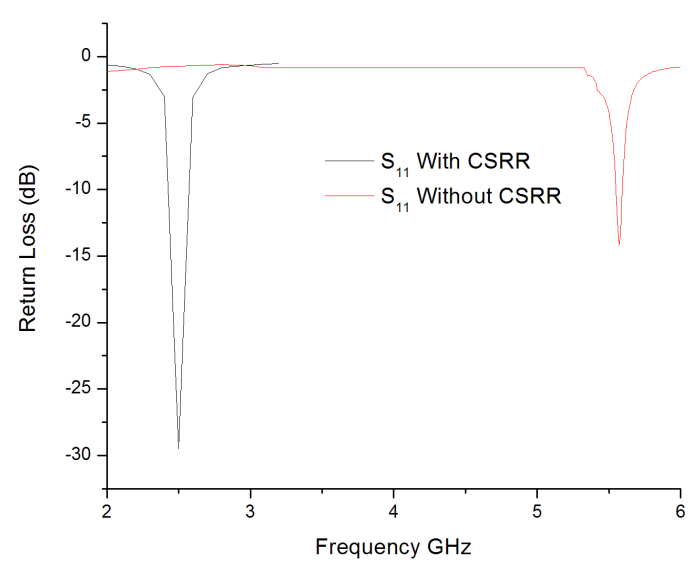

Figure 3: Comparison of reflection coefficient with and without CSRR loaded patch

\subsection{Return Loss and Isolation for MIMO Antenna}

Fig. 6 shows the MIMO antenna system with CSRR loaded patches. Fig. 4, 5 shows the comparative plot for simulated and measured return loss and isolation characteristics. There is a slight shift in the resonant frequency from $2.5 \mathrm{GHz}$ to $2.7 \mathrm{GHz}$ compared to the simulation result due to fabrication losses. The simulation results for the return loss were observed to be $-26.5 \mathrm{~dB}$ at the resonant frequency of 2.5 $\mathrm{GHz}$ and $-38 \mathrm{~dB}$ during measurement at the resonant frequency of $2.7 \mathrm{GHz}$. The simulation result for the isolation was observed to be $-17.3 \mathrm{~dB}$ at the resonant frequency of 2.5 $\mathrm{GHz}$ and the measured result for the isolation is $-15.1 \mathrm{~dB}$ at the resonant frequency of $2.7 \mathrm{GHz}$, as shown in Fig. 5. The designed antenna covered a bandwidth of 2.42 to $2.57 \mathrm{GHz}$ using simulation whereas 2.64 to $2.74 \mathrm{GHz}$ for the measurement.

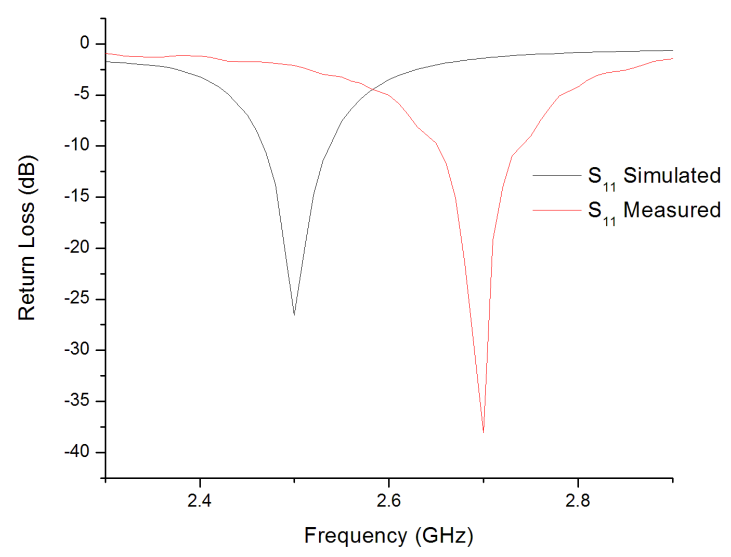

Figure 4: Simulated and measured return loss (dB) 


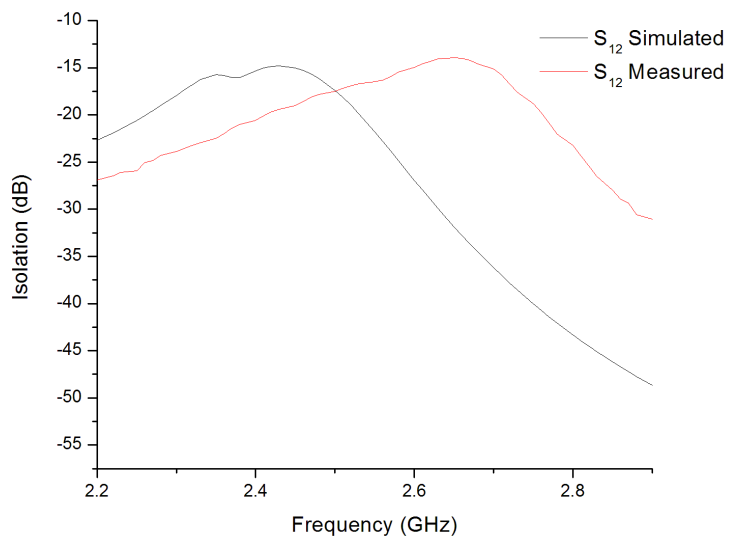

Figure 5: Simulated and measured Isolation (dB)

The proposed antennas described in the previous section were first designed and tuned in HFSS. They were then fabricated for the MIMO configuration as shown in Fig.6 (a), (b) showing the top and bottom layer.

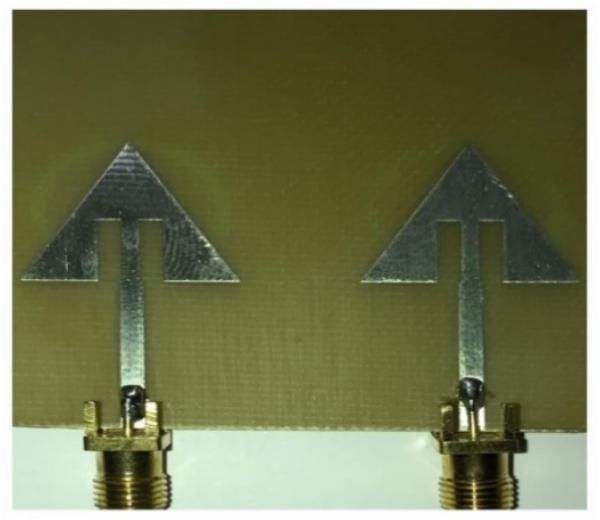

Figure 6: Fabricated antenna (a) Top layer.

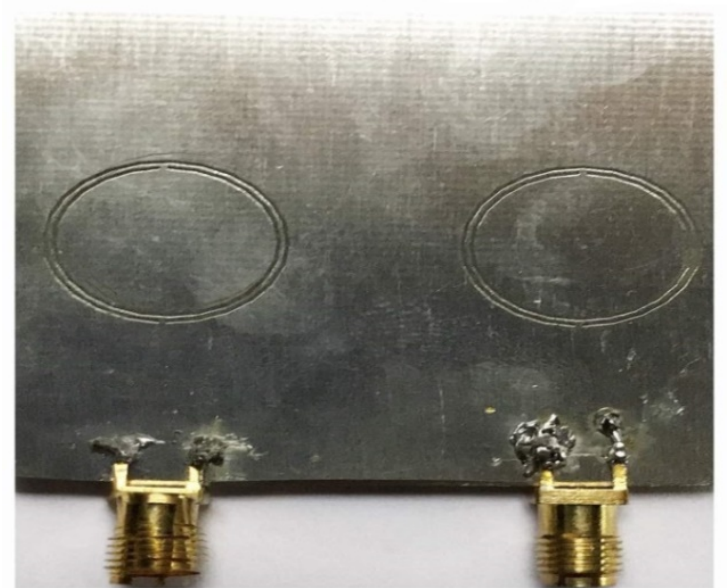

Figure 6: Fabricated antenna (b) bottom layer.

\subsection{MIMO Performance Parameters}

To properly characterize the efficiency and bandwidth of the MIMO antenna system, the scattering matrix is not enough [24].Thus, for better characterization of the MIMO antenna system, the total active reflection coefficient (TARC) and correlation co-efficient are computed.Total Active Reflection Coefficient (TARC) is defined as the square root of the ratio of the sum of the power available at all the ports minus the radiated power to the total available power [25]. It is a real number between 0 and 1 [26]. When its value is zero, this means that all the available power is radiated. The proposed antenna has a TARC value of 0.56 which is within 0 to 1 as shown in Fig. 7.

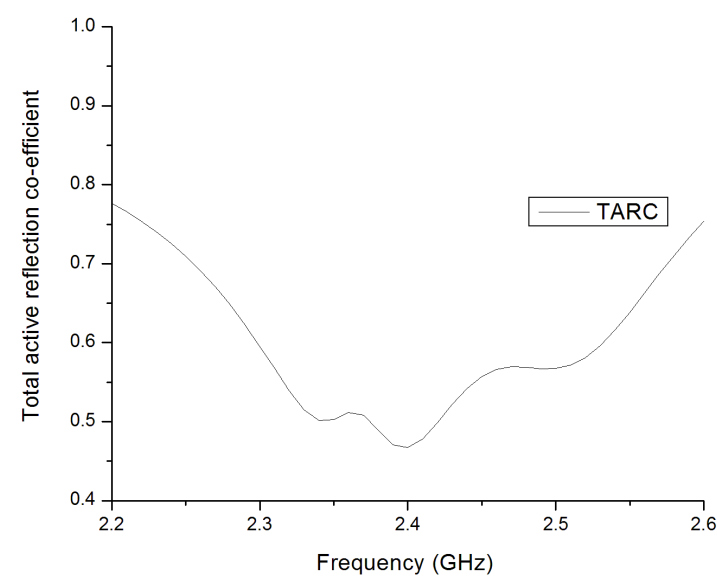

Figure 7: Simulated total active reflection co-efficient

The correlation coefficient $\rho$ is a measure that describes how the communication channels are isolated or correlated with each other. High isolation and low correlation coefficients are required for a MIMO antenna system to provide good diversity performance [25]. The square of the correlation coefficient is the Envelope Correlation Coefficient (ECC). The correlation coefficient can be computed by using $S$ parameters. An envelope correlation coefficient value of 0.5 has been set as an acceptable value for diversity conditions .The proposed LTE-MIMO provides the envelope correlation coefficient below 0.0212 over the LTE frequency band as shown in Fig.8. 


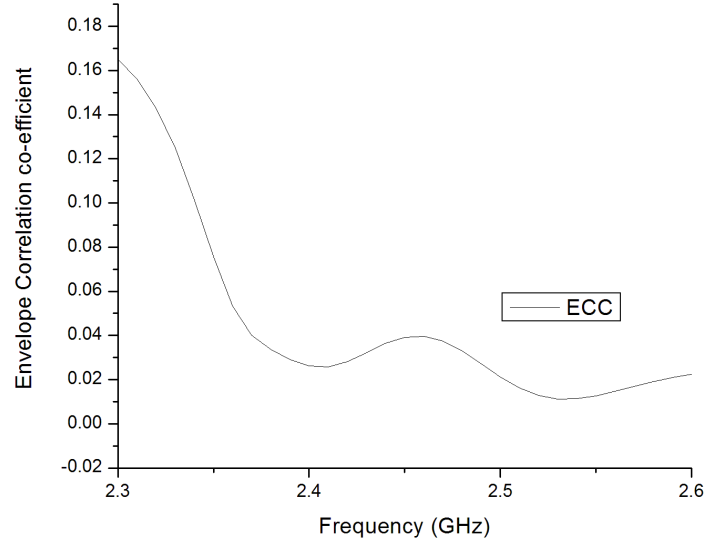

Figure 8: Simulated envelope correlation co-efficient

The realized gain was found to be $0.2148 \mathrm{~dB}$ at the resonating frequency of $2.5 \mathrm{GHz}$ and is shown in Fig. 9.

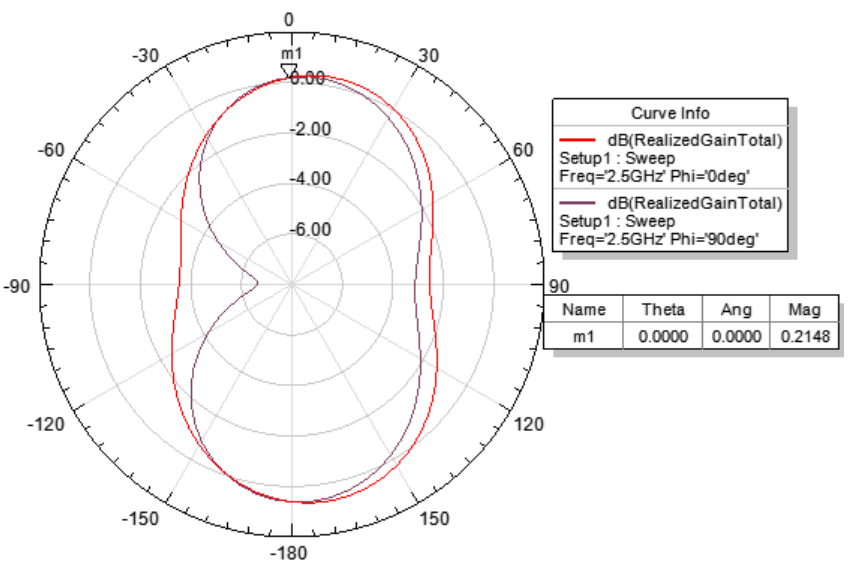

Figure 9: Simulated Realized Gain

The realized gain gets reduced to $-29 \mathrm{~dB}$ without CSRR.

\section{Conclusion}

In this paper, compact $2 \times 1$ MIMO antenna system was presented. The antenna was fabricated on FR4 substrate and occupied a total size of $50 \times 50 \times 0.8 \mathrm{~mm}^{3}$. Antenna miniaturization of $72 \%$ was achieved by loading the patches with CSRRs. The antenna was characterized for MIMO antenna parameters such as TARC and correlation coefficient. The MIMO antenna elements had good isolation thus good diversity performance. Due to its compact size and good performance, the design can be easily employed in a number of wireless portable devices operating in the $(2.5$ 2.57) $\mathrm{GHz}$ LTE band 7 operation.

\section{References}

[1] Garg, V. K., Wireless Communications and Networking, Elsevier-Morgan Kaufmann, Waltham, MA, 2007.

[2] Sesia, Stefania, Matthew Baker and Issam Toufik. LTEthe UMTS Long Term Evolution: From Theory to Practice. Chichester, U.K.: Wiley, 2009.

[3] Zhang, Y. and B. Niu, "Compact Ultra Wideband (UWB) Slot Antenna with Wideband and High Isolation for MIMO Applications", Progress in Electromagnetics Research C, Vol. 54, 9-16, 2014.

[4] Schaubert. D. and K. Yngvesson, "Experimental Study of a Microstrip Array on High Permittivity Substrate", IEEE transactions on Antennas and Propagation, Vol.34, No. 1, 92-97, 1986.

[5] Herscovici, N., M. F. Osorio, and C. Peixeiro, "Miniaturization of Rectangular Microstrip Patches using Genetic Algorithms", IEEE Antennas and Wireless Propagation Letters, Vol. 1, No.1, 94-97, 2002.

[6] Bokhari, S. A., et al., "A Small Microstrip Patch Antenna with a Convenient Tuning Option", IEEE Transactions on antennas and Propagation, Vol. 44, No. 11, 1521-1528, 1996.

[7] Latif, S. I., L. Shafai, and C. Shafai, "An Engineered Conductor for Gain and Efficiency Improvement of Miniaturized Microstrip Antennas", IEEE Antennas and Propagation Magazine, Vol. 55, No. 2, 77-90, 2013.

[8] Chow, Y. L., K. L. Wan, and T. K. Sarkar, "Patch Antenna Miniaturizing with a Shorting Pin Near the Feed Probe-its Physical Mechanism and the Design on Smith Chart", Asia- Pacific Microwave Conference, APMC 2001, Vol. 3, IEEE, 2001.

[9] Kuo, J.-S. and K.-L. Wong, "A Compact Microstrip Antenna with Meandering Slots in the Ground Plane", Microwave and Optical Technology Letters, Vol. 29, No. 2, 95-97, 2001.

[10] Huang, J., "The Finite Ground Plane Effect on the Microstrip Antenna Radiation Patterns", IEEE Transactions on Antennas and Propagation, Vol. 31, No. 4, 649-653, 1983.

[11] Liu, J., W.-Y. Yin, and S. He, "A New Defected Ground Structure and its Application for Miniaturized Switchable Antenna", Progress In Electromagnetics Research, Vol. 107, 115-128, 2010.

[12] Khan, M. U., M. S. Sharawi and R. Mittra, "Microstrip Patch Antenna Miniaturisation Techniques: a Review", IET Microwaves, Antennas \& Propagation, Vol. 9, No. 9, 913-922, Jun. 2015.

[13] Dong, Y., H. Toyao, and T. Itoh, "Design and Characterization of Miniaturized Patch Antenna Loaded with Complementary Split Ring Resonators", IEEE Transactions on Antennas and Propagation, Vol. 60, No. 2, 772-785, Feb.2012.

[14] Ouedraogo, R. O., E. J. Rothwell, A. R. Diaz, K. Fuchi, and A. Temme, "Miniaturization of Patch Antennas using a Metamaterial-Inspired Technique", IEEE Transactions on Antennas and Propagation, Vol. 60, No. 5, 2175 - 2182, May 2012. 
[15] Khan, M. U. and M. S. Sharawi, "A Compact 8Element MIMO Antenna System for 802.11ac WLAN Applications", International Workshop on Antenna Technology, (IWAT), 91-94, 2013.

[16] Asieh, H., J. Nouriniaand C. Ghobadi, "Mutual Coupling Reduction Between Very Closely Spaced Patch Antennas using Low-Profile Folded SplitRing Resonators (FSRRs)", IEEE Antennas and Wireless Propagation Letters, Vol. 10, 862 - 865, IEEE, 2011.

[17] Saraswat, R. K. and M. Kumar, "Miniaturized Slotted Ground UWB Antenna Loaded with Metamaterial for WLAN And WiMAX Applications", Progress In Electromagnetics Research B, Vol. 65, 65-80, 2016.

[18] Baena, J. D., J. Bonache, F. Martin, R. Marques, F. Falcone, T. Lopetegi, M. A. G. Laso, J. Garcia, I. Gil, and M. Sorolla,, Equivalent-Circuit Models for SplitRing Resonators and Complementary Split Ring Resonators Coupled to Planar Transmission Lines", IEEE Transactions on Microwave Theory and Techniques, Vol. 53, No. 4, 1451-1461, Apr. 2005.

[19] A. Christina Josephine Malathi and D.Thiripurasundari, "Review on Isolation Techniques in MIMO Antenna Systems", Indian Journal of Science and Technology, 9.35, 2016.

[20] Cheng, X., D. E. Senior, C. Kim, and Y. Yoon, “A Compact Omnidirectional Self-Packaged Patch Antenna with Complementary Split-Ring Resonator Loading for Wireless Endoscope Applications", IEEE Antennas and Wireless Propagation Letters, Vol. 10, 1532-1535, 2011.

[21] Ziolkowski, Richard W, "Design, Fabrication and Testing of Double Negative Metamaterials", IEEE Transactions on Antennas and Propagation, Vol.51, no.7, pp. 1516-1529, July 2003.

[22] Rothwell, Edward J., et al, "Analysis of the NicolsonRoss-Weir Method for Characterizing the Electromagnetic Properties of Engineered Materials", Progress In Electromagnetics Research, 157 pp.31-47, 2016.

[23] Wirgin, Armand, "Retrieval of the Frequency-

Dependent Effective Permeability and Permittivity of the inhomogeneous Material in a Layer", Progress in Electromagnetics Research B, 70, pp.131-147, 2016.

[24] S. Ho-Chae, S. Oh, and S. Park, "Analysis of Mutual Coupling, Correlations, and TARC in WiBro MIMO Array Antenna", IEEE Antenna Wireless Propag. Lett., Vol. 6, pp. 122-125, 2007.

[25] Choukiker, Y. K., S. K. Sharma, and S. K. Behera, "Hybrid Fractal Shape Planar Monopole Antenna covering Multiband Wireless Communications with MIMO Implementation For Handheld Mobile Devices", IEEE Transactions on Antennas and Propagation, Vol. 62, No. 3, 1483-1488, Mar. 2014.

[26] Sharawi, M. S., M. U. Khan, A. B. Numan, and D. N. Aloi, "A CSRR Loaded MIMO Antenna System for ISM Band operation", IEEE Transactions on Antennas and Propagation, Vol. 61, No. 8, 4265-4274, Aug. 2013. 